\title{
Ciencia de la información y educación: la formación de los profesores de los profesionales de la información
}

\author{
Mara Eliane Fonseca Rodrigues \\ Universidade Federal Fluminense, Niteroi (Rio de Janeiro, Brasil)
}

\section{Resumen}

En este trabajo se analiza la formación de los formadores de los profesionales de información a partir de las exigencias impuestas por la sociedad contemporánea. El análisis se orienta hacia una cuestión: ¿hasta qué punto la didáctica tradicional puede contribuir, efectivamente, a la formación de un ser humano capaz de responder a los requisitos de la sociedad del conocimiento? Apoyado en las teorías de Santos (1999, 2002a y 2002b) y Morin (2000a y 2000b), que proponen una nueva visión de la sociedad y la educación, este estudio tiene como objetivo aportar pistas para la reflexión acerca de la formación de los profesores de ciencia de la información. El trabajo intenta incorporar el referente teórico utilizado al concepto de "reflexión en la acción" de Schön (1995) y a las ideas de Nóvoa (1995), investigador portugués que estudia el trabajo del profesor. Para esto, en un primer momento, se cuestiona el modelo de enseñanza practicado por las instituciones de enseñanza superior, a partir de una concepción positivista de la ciencia, del conocimiento y del mundo. Seguidamente se analiza el paradigma pedagógico emergente como una combinación de tres conceptos pedagógicos: el enfoque progresista, la enseñanza con investigación y la visión sistémica. A continuación se discute la noción de enseñanza con investigación y se discute si los docentes/investigadores del campo de la ciencia de la información son estimulados durante su formación a trabajar la investigación como un instrumento de enseñanza y un contenido de aprendizaje. Finalmente, se llama la atención sobre la necesidad de que los formadores de profesionales de la información promuevan cambios efectivos en sus practicas pedagógicas de modo que rompan con la linealidad de las didácticas tradicionales con el fin de preparar mejor a los futuros profesionales para que contribuyan a la reestructuración productiva de la sociedad en función del proceso de globalización del mundo contemporáneo.

Palabras clave: Formación de los profesores de ciencia de la información. Enseñanza con investigación. Paradigma emergente.

Scire. $12: 2$ (jul.-dic. 2006) 37-53. ISSN 1135-3716. 


\section{Abstract}

This essai deals with educators of information professionals, in view of requirements imposed by society. The analysis is guided by the following question: in what measure the traditional pedagogical methods, effectively, contribute to the education of qualified professionals to act in knowledge society? Based on the theories of Santos (1999, 2002a and 2002b) and Morin (2000a and 2000b), who propose a new perspective of society and education, this research intends to reflect on education of information science professors. This work integrates Shön's theory of "reflection in action" (1995) and the ideas of Nóvoa (1995), a Portuguese researcher especially interested in the study of teaching profession. The research questions the model of university education, based on a positivist vision of science, of knowledge and of the world. It analyzes the proposal of the emergent paradigm, anchored in pedagogical practice that makes possible the production of knowledge, points to the building of an alliance between three pedagogical concepts: the progressive concept, the education with research and the systemic vision. It focuses the conception of education with research and argues if professors and researchers in the area of information science are stimulated to practice research as an instrument of education during their education. Finally, it underlines the importance of the professors of information professionals to promote changes in their pedagogical practices trying to modify their traditional models of education in order to prepare professionals to take care of the reorganization of contemporaneous society.

Keywords: Education of information science professors. Education with research. Emergent paradigm.

\section{Introducción}

En las dos últimas décadas del siglo XX hemos asistido a grandes transformaciones tanto en el campo socioeconómico y político como en el de la cultura, la ciencia y la tecnología. Estos cambios, que se produjeron de forma acelerada y contundente, modificaron las expectativas y necesidades de los hombres. Al principio de un nuevo siglo, vivimos un tiempo de esperas, de perplejidad, de crisis de concepciones y paradigmas.

Hoy la sociedad demanda un hombre que no solo sepa transitar por los meandros de un área específica, sino que no se inhiba ante la inseguridad y la velocidad de las transformaciones tecnológicas. El mundo contemporáneo, globalizado y altamente técnico, exige personas cada vez más cualificadas, tanto en el plano cultural como en la escolaridad formal. De igual modo, las habilidades exigidas en los profesionales son cada vez más sofisticadas, puesto que la evolución del modo de producción desprecia el trabajo no cualificado y repetitivo. Todas esas alteraciones requieren una propuesta renovadora por parte del sistema educativo que per- 
mita poner en práctica nuevas ideas, nuevos ideales y nuevas experiencias de educación. En este sentido, la enseñanza superior, debido a su capacidad para transformar e inducir cambios y adelantos en la sociedad, se considera un componente esencial.

La enseñanza superior está reconocida en cualquier sociedad como un motor de desarrollo y, a la vez, como el principal instrumento para acceder a la experiencia cultural y científica acumulada por la humanidad. Las universidades, entendidas como centros autónomos de investigación y creación del saber, son consideradas las responsables del cumplimiento de los objetivos de la enseñanza superior y de la difusión de sus valores fundamentales; en particular tienen la misión de contribuir al desarrollo sostenible del país y a la mejora del conjunto de la sociedad.

La importancia del papel que la enseñanza superior desempeña en la sociedad es reconocida a escala mundial. En la Conferencia Mundial sobre Enseñanza Superior organizada en París por la UNESCO en 1998, cuando se intentó identificar las dificultades y los objetivos de cada continente en materia de enseñanza superior, se hicieron las siguientes consideraciones:

En el inicio de un nuevo siglo, existe una demanda sin precedentes y una gran diversificación en la enseñanza superior, así como una mayor conciencia de su importancia vital tanto para el desarrollo sociocultural y económico como para la construcción del futuro, frente al cual las nuevas generaciones deberán estar preparadas con nuevas cualificaciones, conocimientos e ideas. (UNESCO, 1999, p. 17)

Asimismo, el conocido informe Delors (2000), elaborado por la Comisión Internacional de la UNESCO sobre la Educación para el Siglo XXI bajo el título Educacão, un tesouro a descobrir, destaca como condición necesaria para las próximas generaciones cuatro saberes fundamentales: aprender a aprender, aprender a ser, aprender a hacer y aprender a vivir junto $a$.

Esas concepciones definen una nueva visión del papel de la educación: no se trata solo de transmisión de contenidos, sino de formación de sujetos críticos, reflexivos, autónomos y éticos. Con ese nuevo enfoque se espera que los alumnos sean capaces de afrontar situaciones inesperadas, solucionar problemas inéditos, desarrollar el conocimiento en sus áreas de interés y respetar la pluralidad cultural.

Esas expectativas nos hacen pensar en la formación de los formadores de los profesionales de la información y nos llevan a plantearnos una pregunta: ¿hasta qué punto las didácticas tradicionales se muestran todavía capaces de contribuir efectivamente a la formación de un hombre capacitado para responder a las exigencias de la sociedad del conocimiento?

Apoyado en el referente teórico de Santos (1999, 2002a y 2002b) y Morin (2000a y 2000b), que proponen una nueva visión de la sociedad y de la educación, la finalidad del presente trabajo es aportar pistas para la reflexión sobre la forma-

Scire. 12 : 2 (jul.-dic. 2006) 37-53. ISSN 1135-3716. 
ción de los profesores del área de ciencia de la información en relación con las exigencias impuestas por la sociedad contemporánea. El estudio intenta, asimismo, incorporar el referente teórico utilizado al concepto de "reflexión en la acción" de Schön (1995) y a las ideas de Nóvoa (1995), investigador portugués especialmente interesado en el estudio de la profesión docente.

Para ello, en un primer momento se cuestiona el modelo educativo practicado por las instituciones de enseñanza superior, a partir de una concepción positivista de la ciencia, el conocimiento y el mundo. Seguidamente se analiza el paradigma pedagógico emergente como una combinación de tres conceptos pedagógicos: el enfoque progresista, la enseñanza con investigación y la visión sistémica. A continuación se enfoca el concepto de enseñanza con investigación y discute si los docentes/investigadores del área de ciencia de la información son estimulados durante su formación a trabajar la investigación como un instrumento de enseñanza y un contenido de aprendizaje. Finalmente, partiendo de las discusiones formuladas a lo largo del trabajo, se llama la atención sobre la necesidad de que los formadores de profesionales de la información promuevan cambios efectivos en sus practicas didáctico-pedagógicas de modo que rompan con la linealidad que prevalece en las didácticas tradicionales con el fin de preparar mejor los futuros profesionales para que contribuyan a la reestructuración productiva de la sociedad en función del proceso de globalización del mundo contemporáneo.

\section{Las nociones de conocimiento, ciencia y mundo y la enseñanza en la universidad}

La enseñanza impartida en las universidades se basa en la tradición cultural del pensamiento hegemónico occidental que Santos (2002b) denomina paradigma dominante. Este paradigma se instauró a partir del siglo XVI con la revolución científica, gracias a las ideas de Copérnico, Galileo, Descartes y Newton, "constituyendo un orden que admite una única forma de conocimiento verdadero denominado conocimiento científico, una única racionalidad, la razón cognitiva instrumental" (Fernández, 2001, p. 178).

Galileo destaca como el gran genio de su época: confirma las ideas de Copérnico e introduce la descripción matemática de la naturaleza y el razonamiento empírico, que se convirtieron en las características predominantes del pensamiento científico del siglo XVII. Con él tiene lugar el verdadero cambio científico hacia la inducción y la deducción, a partir de las cuales se procesa el conocimiento.

Descartes, considerado como el padre del racionalismo moderno, sistematiza el nuevo orden histórico que busca controlar la naturaleza y las fuerzas del universo. Descartes establece las bases del método racional-deductivo, teniendo la duda como punto fundamental de su método y utilizándola como instrumento básico de raciocinio, prescindiendo de la demostración. La razón para Descartes es algo ab- 
soluto y antecede a cualquier objeto. Su método analítico propone la descomposición del pensamiento y de los problemas en sus partes componentes y la disposición dentro de un orden lógico. Su concepción de la naturaleza se asienta en dos dominios independientes y separados: mente y materia.

Newton complementa el pensamiento de Descartes haciendo realidad la visión del mundo como máquina perfecta. La imagen del universo que el modelo de Newton aporta es la de un sistema mecánico susceptible de ser descrito objetivamente y no en relación con el observador humano, un sistema que actúa como una máquina gobernada por leyes inmutables. De acuerdo con Santos (2002b, p. 17), "esta idea de mundo-máquina es tan poderosa que se va a transformar en la gran hipótesis universal de la época moderna, el mecanicismo". Esa visión va a determinar una nueva relación de la humanidad con la naturaleza, una relación mecanicista, externa, que sustituye a la basada en símbolos y mitos construidos culturalmente.

Ese paradigma ha guiado a la sociedad moderna, y en especial a la escuela, en todos los niveles de la enseñanza. Como consecuencia del pensamiento cartesianonewtoniano, las escuelas repartieron el conocimiento en áreas, las áreas en cursos, los cursos en asignaturas, las asignaturas en especialidades (Behrens, 1999, p. 384). Otro factor relevante de influencia de ese paradigma en la relación docente es la búsqueda de reproducción del conocimiento.

Caracterizada por la fragmentación, la práctica pedagógica propone acciones mecánicas a los alumnos, provocando una enseñanza asentada en el escuche, lea, memorice y repita. Estas cuatro acciones han sido propuestas como metodología en la enseñanza universitaria durante un largo período en la historia de la educación. (Behrens, 1999, p. 394)

Ese paradigma epistemológico, con sus principios y leyes, definió la enseñanza en la universidad, y esa es la lógica mediante la cual el profesor está llevando a cabo su formación, incluso en el área de la ciencia de la información. Sin embargo, la transición que estamos viviendo a partir de la teoría de la relatividad y de los nuevos descubrimientos en la física cuántica y sus implicaciones en la filosofía de la ciencia ofrece posibilidades de múltiples racionalidades caracterizadas por Santos (2002b) como paradigma emergente y por Morin (2002b) como pensamiento complejo. Estas propuestas están generando nuevas formas de concebir y producir la ciencia y el conocimiento, y de actuar en el mundo y modificarlo. Sostienen un principio unificador del saber y del conocimiento en torno al ser humano, valorando su cotidianidad, su singularidad y su entorno, entre otros aspectos. Creen en la necesidad de la (re)construcción del hombre y del mundo teniendo la educación entre sus pilares fundamentales.

Por todo ello, a continuación analizamos estas proposiciones y su posible impacto en la práctica pedagógica en la universidad.

Scire. $12: 2$ (jul.-dic. 2006) 37-53. ISSN 1135-3716. 


\section{El paradigma educacional emergente y su impacto en la práctica pedagógica en la universidad}

En las propuestas de Santos y en las de Morin está presente la posibilidad de una nueva matriz para construir la ciencia que sobrepase el pensamiento epistemológico positivista y presente nuevas alternativas para idear el proceso enseñanzaaprendizaje en la universidad.

Santos (1999, 2002a y 2002b), al analizar la trayectoria de la ciencia moderna, anuncia que el modelo de racionalidad que la preside se encuentra en crisis. Considera este autor que a partir de esta ruptura del equilibrio se origina una nueva perspectiva para hacer ciencia denominada paradigma emergente. Este paradigma propuesto por Santos, basado en la teoría de la relatividad y en la física cuántica, busca una nueva lectura del mundo y una manera diferente de posicionarse el hombre en él, partiendo de un tipo de pensamiento que aborda las cosas en conjunto. En él están presentes todas las contradicciones que el paradigma tradicional niega, admitiendo la no neutralidad del conocimiento, reconociendo la intencionalidad del sujeto y concibiendo la ciencia como un acto humano históricamente situado. Este paradigma, de acuerdo con Santos (2002b), puede ser explicitado a través de los siguientes principios: $a$ ) todo conocimiento científico-natural es científico-social, lo que quiere decir que el contenido teórico de las ciencias está cada vez más regido por conceptos, teorías, metáforas y analogías de las ciencias sociales; $b$ ) todo conocimiento es total y local: rompe la estructura formal de la disciplinariedad y se compone de temas; $c$ ) todo conocimiento es autoconocimiento, porque siempre es reinterpretado por aquel que lo produce; $d$ ) todo conocimiento científico aspira a constituirse en sentido común para poder dar sentido a la vida.

Partiendo de este nuevo paradigma, el enfoque de la ciencia moderna que analiza el mundo en partes independientes ya no funciona. La nueva perspectiva apunta hacia la (re)construcción del hombre y del mundo teniendo como base una concepción más variada y compleja del saber humano, que es visto como multiforme y no como monolítico. Ese paradigma concibe la ciencia como más transformadora que acumulativa, al contrario de como lo hacía el paradigma tradicional.

Morin (2000a y 2000b) apunta la necesidad de un cambio de perspectiva ante la fragmentación de un mundo que ya se encuentra en un nuevo milenio, con la preocupación por la conciliación de la humanidad con el cosmos no a partir de la síntesis y de la reducción, sino de la amplitud del pensamiento y de las acciones (el pensamiento complejo). Respecto a la educación, Morin (2000a) expresa la idea de que cualquier reforma educativa deberá, antes de nada, empezar por una reforma de los educadores, y que la enseñanza solo puede ser viable si tiene como finalidad la educación integral del ser humano. 
Morin (2000b) considera que hay siete saberes fundamentales que la educación del futuro debería tratar en cualquier sociedad y en cualquier cultura, teniendo en cuenta sus características específicas:

1. Las cegueras del conocimiento: el error y la ilusión. Se ha de enseñar la ciencia como un conocimiento en construcción y sujeto al error y a la ilusión.

2. Los principios del conocimiento pertinente. Hay que promover un conocimiento capaz de aprehender problemas globales y fundamentales para insertar en ellos los conocimientos parciales y locales; enseñar métodos que permitan establecer relaciones mutuas e influencias recíprocas entre las partes y el todo en un mundo complejo.

3. La condición humana. Se debe estimular el conocimiento de la naturaleza compleja del ser humano y la conciencia de su identidad común a todos los otros humanos. Para ello hay que empezar a comprender al hombre como un ser a la vez físico, biológico, psíquico, cultural, social e histórico.

4. La identidad terrena. Se ha de mostrar cómo todas las partes del mundo se volvieron solitarias, aunque sin ocultar las opresiones y la dominación que han devastado la humanidad y que aún no han desaparecido.

5. Las incertidumbres. Hay que enseñar principios de estrategia que permitan afrontar los imprevistos, lo inesperado y la incertidumbre, y modificar su desarrollo en virtud de la información adquirida a lo largo del tiempo.

6. La comprensión. Esta es a la vez medio y fin de la comunicación humana, por lo que debemos considerar la importancia de la educación para la comprensión, en todos los niveles educativos y a todas las edades.

7. La ética del género humano. La ética debe formarse en las mentes a partir de la conciencia de que el ser humano es a la vez individuo, parte de la sociedad y parte de la especie.

Por lo que hemos visto hasta aquí, queda claro que las ideas de Santos y Morin convergen hacia un mismo lugar: la necesidad de cambios. No se trata solo de cambios en tanto que diferencias con respecto al paradigma aún vigente, sino principalmente en relación con la acción de la escuela.

Ambas propuestas buscan vencer la fragmentación generada por el modelo newtoniano-cartesiano y llevan a la necesidad de replantearse la escuela (en su concepción más general). La escuela pasa a ser vista como articuladora del conocimiento: no es solo un espacio físico, "sino un estado permanente del individuo, donde el trabajo en colaboración está siempre presente" (Behrens, 1999, p. 387). El presupuesto esencial de esas propuestas es una práctica pedagógica que posibilite la producción del conocimiento y permita una unión de enfoques y tendencias pedagógicas que atiendan a la demanda de la sociedad del conocimiento o de 
la información apuntando hacia un nuevo paradigma educativo: el paradigma educacional emergente.

En este sentido, Moraes (1996, pp. 123-165 y 179-187), en su tesis doctoral, que propone un paradigma educativo capaz de fundamentar un nuevo proyecto pedagógico para la escuela brasileña, considera que el paradigma emergente probablemente ocasionará alteraciones en la acción de la escuela, afectando a la misión de la misma, al eje del trabajo docente, al objeto de la labor educativa, a la relación entre los sujetos involucrados en esta labor y a la percepción del contexto educacional. En cuanto al contexto educacional, contiene, desde la perspectiva de este paradigma, ciertas propiedades: relacional, interaccional, constructivista, sociocultural, trascendental, reflexiva, autonomista, cooperativa, crítica y ciudadana. Estas propiedades apuntan a situaciones colectivas y aportan al contexto concreto de la educación la redefinición de los papeles de profesor y alumno en el espacio de la escuela. El primero pasa a tener "un papel fundamental de articulador y mediador entre el conocimiento elaborado y el conocimiento que se ha de producir" (Behrens, 1999, p. 386), y también pasa a ser visto como un aprendiz. El alumno es considerado igualmente un enseñante, pues se lo ve como un sujeto activo. Así, el eje del trabajo docente deja de ser la enseñanza y pasa a ser el aprendizaje: alumnos y profesores se encuentran unidos en procesos de aprendizaje verdaderamente interactivos, trabajando en la construcción de un todo comprensible de conocimiento a partir de hechos contextualizados.

Esta noción se presenta como posible en cualquier nivel escolar y gana más vigor en la esfera de la enseñanza universitaria. Ya discutimos anteriormente que los principios epistemológicos y las reglas metodológicas que constituyen el paradigma dominante definen la forma y el contenido de la enseñanza en la universidad. En la próxima organización de los currículos de los cursos de graduación en Brasil se puede observar esta tendencia: se parte de lo teórico hacia lo práctico, disociándose teoría y práctica por una fragmentación disciplinar. El presupuesto, en este modelo, es que primero el alumno tiene que aprender los contenidos generales (absorbiendo el conocimiento ya producido), para después intentar aplicar o reconocer la aplicación de estos contenidos a la realidad. De ahí el énfasis en métodos basados en la repetición y la memorización de relaciones. Dentro de esa lógica, el conocimiento se estructura predominantemente como un saber acabado, descontextualizado y ahistórico. El sistema de evaluación de la enseñanza-aprendizaje, con una posición conceptual basada en certezas, se orienta hacia las cuantificaciones y los conceptos memorizados; esto es resultado de un trabajo que da poca importancia a procesos mentales superiores tales como la comparación, el juicio, el análisis de hipótesis y la creación o resolución de problemas. Esos procedimientos quedan reservados a los asistentes a los cursos de posgrado. Por lo tanto, lo que vemos en la organización actual de los currículos brasileños es un modelo de enseñanza 
que hace hincapié en la repetición y en la memorización y donde el alumno es inducido a no cuestionar, a seguir un camino único en su aprendizaje, basado en el conocimiento transmitido por el profesor.

Pero, según hemos visto anteriormente, el paradigma educacional emergente aporta al contexto concreto de la enseñanza el que el profesor sea considerado también como un aprendiz y el alumno lo sea a su vez como un enseñante. En el caso del primero, su papel de transmisor de información se transforma en el de mediador pedagógico que está junto a los alumnos: pasa de profesor enseñante a profesor que está con el alumno, desarrollando un trabajo en equipo donde él y sus alumnos son agentes/compañeros y corresponsables en las acciones de aprendizaje, o sea, se responsabilizan de la búsqueda de información, aprenden a localizarla, a analizarla y a relacionar los nuevos datos con los conocimientos anteriores para darles significado propio, elaboran conclusiones, observan situaciones de campo y las registran, trabajan con esos datos y tratan de encontrar solución a los problemas, entre otras actividades (Masetto, 2001).

Antônio Nóvoa, en una entrevista concedida en Brasil durante su participación en el I Congresso Ibero-Americano de Formação de Professores, organizado por la Universidade Federal de Santa Maria, en el estado de Rio Grande do Sul, argumenta que la universidad, por medio de las transformaciones de la sociedad actual, "tiene que organizarse y pasar de la función transmisora del conocimiento a funciones de reconstrucción, de crítica y de producción de nuevos conocimientos". Espera que las universidades concedan cada vez más atención al acompañamiento de los alumnos, a través de "formas de orientación y tutorías de orientación e integración de los alumnos en grupos de investigación". A su entender, "será ese conjunto de actividades pedagógicas y científicas, y no las clases propiamente dichas la que definirá la universidad del futuro" (Nóvoa, 2000, p. 132; la cursiva es nuestra).

Aquí se hace importante destacar el concepto de clase en la universidad brasileña. Tradicionalmente la clase se ha constituido en un espacio físico y un tiempo determinado durante el cual el profesor transmite datos y experiencias a un grupo de alumnos sin que estos tengan necesariamente unos conocimientos previos sobre ellos. Generalmente, el modelo de enseñanza empleada se basa en que quien sabe - el profesor - enseña a quien no sabe —el alumno.

Sin embargo, Masetto (2001, p. 85) considera que "dondequiera que haya un aprendizaje significativo que busque intencionadamente alcanzar objetivos definidos, ahí encontramos una clase universitaria". Esta concepción hace que el aula trascienda la idea habitual de algo que acontece solamente en un determinado horario y espacio físico. Otros lugares donde se llevan a cabo, por ejemplo, las actividades profesionales del estudiante — empresas, laboratorios de informática, bibliotecas, centros de información, Internet, entre muchos otros- son reconocidos como espacios en los que este puede acceder a un aprendizaje significativo del 
ejercicio competente de una profesión. En ese sentido, clase significa vivencia, o sea, integración con la realidad.

Hemos visto que la propuesta de una práctica pedagógica que atienda a la producción del conocimiento, y no solo a su transmisión, constituye un gran desafío para los profesores universitarios, ya que afecta al origen mismo de su acción pedagógica: de transmisores de informaciones pasan a ser mediadores pedagógicos, para desarrollar un trabajo en colaboración con los alumnos, donde unos y otros son compañeros y corresponsables en las acciones de enseñanza-aprendizaje.

En lo que respecta al profesor del área de ciencia de la información, en Brasil ese desafío se hace aún mayor debido a las características propias de la enseñanza en esa área. De un modo general, la formación del profesional de la información está relacionada

con un modelo de enseñanza transferidor de conocimientos, asociado a manuales, libros didácticos, sistemas e instrumentos de gestión de información que son tomados más como objetos que han de ser revisados en su estado final que como objetos que deben ser revisados críticamente como un saber transitorio y susceptible de asimilación y transformación en el medio al que va destinado. (Souza, 2004, p. 16)

Sobre todo en el caso de los docentes brasileños que se dedican a la formación de los profesionales de la información, la propuesta contenida en el paradigma educacional emergente puede ayudar a superar las lagunas existentes en un área tradicionalmente relacionada con el hacer más que con el pensar para llegar a la efectiva producción/construcción del conocimiento, aportando un aire de renovación a la enseñanza y a la propia área.

La propuesta del paradigma educacional emergente apunta hacia la combinación de tres conceptos pedagógicos: el enfoque progresista, la enseñanza con investigación y la visión sistémica. El punto de encuentro de estos conceptos es la superación de la repetición y la búsqueda de la producción de conocimientos. Entre ellos, en nuestra opinión, la idea de enseñanza basada en la investigación como elemento constituyente es el punto de partida para redirigir la actual acción docente del profesor universitario y del profesor constructor de conocimientos (Rodrigues, 2002). De esta forma, a continuación vamos a discutir las posibilidades de articulación entre la enseñanza y la investigación, así como la importancia de trabajar ese enfoque en la enseñanza del área de ciencia de la información.

\section{La perspectiva de la enseñanza articulada con la investigación}

Frente a la emergencia de un nuevo paradigma educacional que irremediablemente tenderá a sacudir sus estructuras, la universidad brasileña empieza a plantearse la calidad de las prácticas pedagógicas que en ella se desarrollan. Busca nuevos métodos de enseñanza-aprendizaje y amplía el debate sobre el perfil del profesional que pretende formar.

Scire. 12 : 2 (jul.-dic. 2006) 37-53. ISSN 1135-3716. 
En la actualidad, la preocupación esencial de todas las discusiones que conciernen a las reformas realizadas y a las que están por realizar en la enseñanza universitaria es la formación de unos ciudadanos del mañana con capacidad de autonomía y de crítica, ciudadanos polivalentes, creativos, capaces de distinguir los múltiples desafíos que el siglo XXI indudablemente traerá. Por eso se reconoce que la enseñanza deber ser

una actividad que no se limite a mostrar lo que está hecho, a transmitir el conocimiento acumulado; también, [debe] orientar a las personas para que consigan la información necesaria para solucionar los problemas que la vida les plantea, ya sean cotidianos, profesionales o sociales. (Chizzotti, 2001, p. 106)

Generalmente, la propuesta emergente significa añadir al rigor científico la crítica, la duda epistemológica y la interdisciplinariedad del conocimiento. En esta proposición la duda está en la base del proceso enseñanza-aprendizaje, y por tanto se busca una enseñanza basada en la investigación, que incorpore los procesos metodológicos de esa actividad y tenga la indagación y la duda científica como referencias pedagógicas (Cunha, 1997). Mediante este procedimiento se intenta dotar al alumno de los instrumentos necesarios para que actúe con mayor independencia intelectual.

La investigación puede ser considerada como "un esfuerzo metodológico de búsqueda de información para producir conocimientos nuevos, ampliar la comprensión del mundo y ayudar a solucionar los problemas concretos que las personas han de afrontar" (Chizzotti, 2001, p. 106). Aborda preguntas no respondidas, inquietudes que surgen de la práctica o de la reflexión; esto debe convertirse en una actitud cotidiana.

Masetto (2003) considera que la enseñanza con investigación es una estrategia fundamental para mejorar la calidad de la enseñanza universitaria, y es la que ya se está iniciando en la formación de los profesionales contemporáneos. Según el autor, esta estrategia didáctico-pedagógica permite el desarrollo de varios aprendizajes: $a$ ) desarrollar la iniciativa en la búsqueda de informaciones, datos y materiales necesarios para el estudio; $b$ ) entrar en contacto con las más variadas fuentes de información (libros, periódicos, anales de eventos científicos, músicas, fotos...), con los más diversos ambientes informativos (bibliotecas, Internet, sitios web...) y con especialistas del mismo curso y/o área y de otras instituciones mediante entrevistas, correo electrónico, listas de discusión, etcétera; $c$ ) seleccionar, organizar, comparar, analizar y relacionar datos e informaciones; $d$ ) hacer deducciones a partir de datos e informaciones, formular hipótesis, analizarlas, comprobarlas, reformularlas y sacar conclusiones; $e$ ) elaborar informes con características científicas; f) comunicar los resultados obtenidos con claridad, orden y precisión científica, tanto de forma oral como por escrito (Masetto, 2003, p. 104).

Scire. 12 : 2 (jul.-dic. 2006) 37-53. ISSN 1135-3716. 
Sin embargo, es importante señalar que difícilmente el alumno incluirá la investigación en su proceso de aprendizaje si el profesor no lo hace, o sea, si el profesor no aprende también a actualizar y/o renovar sus conocimientos por medio de la investigación, las lecturas, las reflexiones personales, la participación en eventos científicos, etcétera. En lo tocante a los profesores de ciencia de la información brasileños, esa alerta es fundamental, pues, como ya hemos visto, el modelo de enseñanza en esa área es más repetitivo que creativo.

Nóvoa (1995) planta la semilla de la investigación en la actividad del profesor cuando habla de la necesidad de "conjugar una formación de tipo clínico, o sea, basada en la articulación entre la práctica y la reflexión sobre esta práctica, y una formación de tipo investigativo, que enfrente a los profesores con la producción de saberes pertinentes" (Nóvoa, 1995, p. 28).

Esa visión de la formación se inspira en los conceptos de "reflexión en la acción” y "reflexión sobre la acción" de Donald Schön (1995). Su visión de la práctica reflexiva describe diferentes modos de estimular a los profesores para que utilicen su propia enseñanza como forma de investigación destinada al cambio de los métodos. Para Schön, la enseñanza debe ser encarada como una forma de investigación y experimentación, haciendo que las teorías en acción de los profesores adquieran una legitimidad que les es negada por el enfoque dominante de la ciencia aplicada. Sin embargo, este autor considera que "en la formación de profesores, las dos grandes dificultades para la introducción de un praticum reflexivo son, por un lado, la epistemología dominante en la universidad y, por otro, su currículo profesional normativo" (Schön, 1995, p. 91), lo que dificulta la formación de un profesor para que sea más capaz de reflexionar sobre su práctica y su aplicabilidad. Observa que un posible camino para minimizar esa dificultad es incrementar las iniciativas que ya empiezan a emerger y estimular la formación continua de los profesores.

Entre estas iniciativas podemos destacar las diversas formas de trabajar la articulación entre enseñanza e investigación en los cursos universitarios. Una de ellas sería la de que la investigación se convierta en el eje o núcleo del curso, en el norte de toda la estructura curricular. Esto se puede traducir en una organización curricular en la que disciplinas y actividades sean planeadas colectivamente con el objetivo de desarrollar habilidades y actitudes de investigación en los alumnos. También puede derivar en el uso de la investigación como mediación, o sea, que las disciplinas y actividades del curso inciten a los alumnos a desarrollar investigaciones que incluyan el análisis del día a día de las instituciones y/o espacios de información, con el fin de acercar a los futuros profesionales a la realidad del área en la que van a actuar.

Todavía existe la posibilidad de que los docentes del curso inserten sus propios temas y proyectos de investigación en los programas de las disciplinas de modo

Scire. 12 : 2 (jul.-dic. 2006) 37-53. ISSN 1135-3716. 
que sus alumnos tengan la oportunidad de discutir los resultados de sus investigaciones, los datos analizados y la metodología utilizada, para que a partir de allí puedan proponer y generar nuevos temas y problemas.

Hay que destacar que la investigación en la enseñanza universitaria debe ir más allá de la iniciativa de incrementar las disciplinas que traten del tema y sus métodos, más bien ha de ser una actitud presente en el cuerpo docente e irradiarse por todo el itinerario curricular que el alumno va a recorrer en la universidad. De esta forma, todas las actividades del currículo, ya sean asignaturas, talleres, seminarios u otras, deben tener como principio metodológico la investigación, estimulando a los universitarios para que no busquen solamente respuestas, sino también la formulación de sus propias cuestiones acerca de los temas estudiados.

En lo que respecta a la práctica pedagógica desarrollada por los profesores del área de ciencia de la información en Brasil, tales exigencias encuentran reflejos significativos en un momento en que se intenta romper con una formación de profesional eminentemente técnico (volcado hacia la racionalidad instrumental, funcional, inmediata, adaptable) para buscar un perfil de naturaleza más interdisciplinaria, que pueda llegar a una mejor comprensión de la ambigüedad y la complejidad del tiempo presente, y de ese modo dar cuenta de una realidad heterogénea en un mundo globalizado, con usuarios más exigentes y con todo un aparato tecnológico en constante perfeccionamiento.

\section{Consideraciones finales}

Según lo que hemos visto a lo largo del presente trabajo, podemos observar que el paradigma educacional emergente genera impactos sobre la práctica pedagógica universitaria, y en particular sobre la enseñanza del área de ciencia de la información.

Desde la perspectiva de ese paradigma, el actual enfoque que analiza el mundo en partes independientes ya no funciona. Se insiste en la necesidad de (re)construcción del hombre y del mundo contando con la educación como uno de los pilares básicos. En este sentido, el presupuesto esencial es que por medio de una práctica pedagógica innovadora se puede llegar a la producción del conocimiento. Esa propuesta constituye un desafío para los profesores universitarios, especialmente para aquellos que actúan en el área de ciencia de la información, teniendo en cuenta que el objeto de estudio y de trabajo del profesional de la información — la información - es un objeto que forma parte de la vida cotidiana de toda la humanidad y está en intensa interacción con la tecnología.

Podemos apuntar un desafío en lo que respecta a los contenidos y metodologías utilizados por los profesores en los cursos del área de la ciencia de la información en Brasil. La ciencia de la información puede ser vista como un área de 
actuación profesional o como un campo intelectual. No obstante, en la discusión educacional se ha dado prioridad al primer enfoque. Esto implica otra discusión, que se refleja en otro ámbito: el espacio o mundo laboral. En este sentido, la ciencia de la información ofrece un ejemplo concreto de transición de una configuración profesional tradicional a otra más actual, vinculada al perfil de un profesional en condiciones de actuar en varios tipos de instituciones y servicios de información. Sin embargo, generalmente, la enseñanza en Brasil se distingue por seguir todavía un modelo tradicional en el que la práctica de la investigación es incipiente. La consecuencia de este proceso es que se forman profesionales considerados de nivel más técnico que científico. De este modo, para atender a los presupuestos del paradigma educacional emergente los cursos tendrán que incluir en sus propuestas pedagógicas contenidos y metodologías de enseñanza adecuadas a las nuevas tareas ocupacionales y profesionales.

Otro desafío que puede ser mencionado, retomando la discusión presentada por Moraes (1996) sobre las alteraciones que el paradigma educacional emergente probablemente ocasionará en la acción de la escuela, tiene que ver con la redefinición de los papeles de profesor y alumno en el espacio de la escuela: profesores aprendices y estudiantes enseñantes. Esto modifica obligatoriamente la pedagogía universitaria, y en particular la del área de ciencia de la información.

Todas estas exigencias nos llevan a hacer referencia a las cuatro perspectivas básicas apuntadas por Pérez (1998, pp. 354-373), las cuales interfieren en el desarrollo práctico de la función docente y de la formación del profesor: $a$ ) la perspectiva académica, $b$ ) la perspectiva técnica, $c$ ) la perspectiva práctica y $e$ ) la perspectiva de reconstrucción social.

La perspectiva académica considera que "la enseñanza es, en primer lugar, un proceso de transmisión de conocimientos y de adquisición de la cultura pública que la humanidad acumuló". En ella, el docente es concebido como un especialista, y su formación estará directamente vinculada al dominio de las disciplinas que componen la cultura y cuyos contenidos debe transmitir.

En la perspectiva técnica, la enseñanza es evaluada por la calidad de los productos y por la eficacia y economía de su realización. En ella, "el profesor es un técnico que domina las aplicaciones del conocimiento científico producido por otros y lo transforma en reglas de actuación". El docente, en esa perspectiva, no necesita llegar al conocimiento científico, solo debe dominar las operaciones de intervención técnica que se derivan de él.

La perspectiva práctica se fundamenta en el presupuesto de que "la enseñanza es una actividad compleja que se desarrolla en escenarios singulares, claramente determinados por el contexto, con resultados en gran parte imprevisibles y cargados de conflictos de valor que requieren opciones éticas y políticas". Por eso, la ex-

Scire. 12 : 2 (jul.-dic. 2006) 37-53. ISSN 1135-3716. 
periencia en el ejercicio de la docencia es fundamental para el profesor: "debe ser visto como un artesano o profesional clínico que ha de desarrollar su sabiduría experimental y su creatividad para afrontar las situaciones únicas, ambiguas, inciertas y conflictivas que configuran la clase". En esta perspectiva la formación del profesor se sostendrá especialmente en el aprendizaje de la práctica, para la práctica y a partir de la práctica.

En la perspectiva de reconstrucción social, "el profesor es considerado un profesional autónomo que refleja críticamente la práctica cotidiana para comprender tanto las características de los procesos de enseñanza-aprendizaje como las del contexto en que la enseñanza ocurre". En esta perspectiva percibimos que se agrupan aquellas posiciones que, incluso con matices diferentes, ven la enseñanza como una actividad crítica y como una práctica social. Dentro de este enfoque, la escuela debe tener capacidad para pensar críticamente sobre el orden social, y el profesor es considerado como un intelectual transformador, comprometido políticamente. Es, al mismo tiempo, educador y activista político, en el sentido de que interviene abiertamente en el análisis y en los debates de los temas públicos.

Según las características mencionadas anteriormente para la enseñanza impartida en el área de la ciencia de la información en Brasil, parece que el docente de esa área se sitúa entre las perspectivas académica y técnica. Luego, para que se pongan en marcha nuevas concepciones y propuestas de enseñanza, no hay que ignorar la cultura profesional del docente y los programas de formación de profesores, que entrañan diferentes matices.

El paradigma educacional emergente aporta a la enseñanza impartida por la universidad, y especialmente en el área de la ciencia de la información, una amplia discusión acerca del trabajo docente y de las exigencias educacionales, sociales y políticas impuestas y que remiten necesariamente a la reestructuración y la mejora de la formación docente. Esas exigencias van desde el uso de las tecnologías de información y comunicación, que implica nuevas formas de comunicación entre profesores y alumnos, hasta el papel de los saberes escolares y la (re)definición de la función social de la escuela. En el caso de los profesores del área de ciencia de la información nos encontramos con una trayectoria que revela las disociaciones teoría/práctica e investigación/enseñanza, lo que denota una enseñanza más inclinada hacia el academicismo y el tecnicismo.

Por eso entendemos que abogar por la investigación como integrante de la enseñanza significa elevar la calidad de esta, de modo que la investigación se convierte en un componente esencial para impulsar una redefinición de la actitud de enseñar y aprender tradicionalmente desarrollada en la universidad, y en especial en el área de la ciencia de la información. 
Para satisfacer las necesidades del alumno — ampliar su horizonte de saber, plantear nuevas cuestiones, preguntar sobre el significado de lo que le ha sido transmitido, reflejar y establecer relaciones nuevas entre las observaciones que hace y los conocimientos que posee, contraponer la información recibida a los resultados de sus prácticas- la enseñanza necesita de la investigación. Entonces, cuando moviliza la curiosidad mueve la búsqueda de información, aclara las dudas y orienta las acciones: la enseñanza pasa a tener un nuevo significado para el alumno.

Hoy, la formación profesional en el área de la ciencia de la información no puede restringirse a la perspectiva de una profesionalización estricta, especializada. Además del dominio de los contenidos inherentes al área, el profesional debe estar preparado para afrontar con competencia y creatividad los problemas derivados de su práctica profesional, así como para producir y difundir conocimientos y para reflejar críticamente la realidad que lo rodea. Por ello se pone de manifiesto la necesidad de una enseñanza dinámica, con docentes que utilicen tecnologías de información y comunicación, que no trabajen de forma aislada, sino en redes académicas nacionales e internacionales, que dominen el conocimiento contemporáneo y lo usen para la resolución de problemas.

Por tanto, nos parece indispensable que se invierta en didácticas que puedan contribuir efectivamente a que la enseñanza en el área de la ciencia de la información se enriquezca con la investigación, siempre que los profesores se comprometan realmente a ayudar a la formación de individuos adaptados a su tiempo, capaces de responder a las exigencias contemporáneas.

\section{Referencias}

Benhrens, Marilda Aparecida (1999). A prática pedagógica e o desafio do paradigma emergente. // Revista Brasileira de Estudos Pedagógicos. 80:196 (set.-dez. 1999) 383-403.

Chizzotti, Antonio (2001). Metodologia do ensino superior: o ensino com pesquisa. // Castanho, Sérgio; Castanho, Maria Eugênia (orgs.). Temas e textos em metodologia do ensino superior. Campinas, SP: Papirus, 2001. 103-112.

Cunha, Maria Isabel (1997). O currículo do ensino superior e a construção do conhecimento. // Fórum Nacional de Pró-Reitores de Graduação. Memória. [Campinas, SP], 1997. 181-185.

Delors, Jacques, et ál. (2000). Educação, um tesouro a descobrir: relatório para a UNESCO da Comissão Internacional sobre Educação para o século XXI. São Paulo: Cortez; Brasilia: MEC/UNESCO. 2000. 4. ${ }^{\mathrm{a}} \mathrm{ed}$.

Fernandes, Cleoni Maria Barboza (2001). Docência universitária e os desafios da formação pedagógica. // Interface - Comunicação, Saúde, Educação. 5:9 (ago. 2001) 177-182. http://www.interface.org.br (2004-07-20).

Masetto, Marcos T. (2001). Atividades pedagógicas no cotidiano da sala de aula universitária: reflexões e sugestões práticas. // Castanho, Sérgio; Castanho, Maria Eugênia

Scire. 12 : 2 (jul.-dic. 2006) 37-53. ISSN 1135-3716. 
(orgs.). Temas e textos em metodologia do ensino superior. Campinas, SP: Papirus, 2001. 83-102.

Masetto, Marcos T. (2003). Competência pedagógica do professor universitário. São Paulo: Summus, 2003.

Moraes, Maria Cândida Borges de (1996). O paradigma educacional emergente. Tese (doutorado em Educação), Pontifícia Universidade Católica de São Paulo. São Paulo, 1996.

Morin, Edgar (2000a). A cabeça bem-feita: repensar a reforma, reformar o pensamento. Río de Janeiro: Bertrand Brasil, 2000.

Morin, Edgar (2000b). Os sete saberes necessários à educação do futuro. São Paulo: Cortez; Brasilia: UNESCO, 2000.

Nóvoa, Antônio (1995). Formação de professores e profissão docente. // Nóvoa, Antônio (coord.). Os professores e a sua formação. Lisboa: Dom Quixote: Instituto de Inovação Educacional, 1995. 15-33.

Nóvoa, Antônio (2000). Universidade e formação docente [entrevista em 18 de abril de 2000]. Interface - Comunicação, Saúde, Educação. 4:7 (ago. 2000) 129-137. http://www.interface.org.br (2004-08-09).

Pérez Gómez, A. I. (1998). A função e formação do professor/a no ensino para a compreensão: diferentes perspectivas. // Gimeno Sacristán, J.; Pérez Gómez, A. I. Compreender e transformar o ensino. Porto Alegre: ArtMed, 1998. 353-379.

Rodrigues, Mara Eliane F. (2002). A formação profissional em biblioteconomia: superando limites e construindo possibilidades. // Encontros Bibli. 13 (maio 2002). http://www.encontros-bibli.ufsc.br (2002-07-03).

Santos, Boaventura de Sousa (1999). Pela mão de Alice: o social e o político na pósmodernidade. São Paulo: Cortez, 1999. 5. ${ }^{\text {e }}$ ed.

Santos, Boaventura de Sousa (2002a). A crítica da razão indolente: contra o desperdício da experiência, vol. 1. São Paulo: Cortez, 2002. 4. ${ }^{\text {a }}$ ed.

Santos, Boaventura de Sousa (2002b). Um discurso sobre as ciências. Oporto: Afrontamento, 2002. 13. ${ }^{\mathrm{a}}$ ed.

Schön, Donald A. (1995). Formar professores como profissionais reflexivos. // Nóvoa, Antônio (coord.). Os professores e a sua formação. Lisboa: Dom Quixote: Instituto de Inovação Educacional, 1995. 77-91.

Souza, Francisco das Chagas de (2004). O modelo educacional emergente e seu impacto sobre a dimensão pedagógica da ciência da informação. Londrina: s. n., 2004. Palestra apresentada no I Encontro Nacional de Educação em Ciência da Informação (I ENECIN), promovido pela Associação Brasileira de Educação em Ciência da Informação (ABECIN), na cidade de Londrina, Paraná, Brasil, de 6 a 8 de julho de 2004.

UNESCO (1999). Conferência Mundial sobre o Ensino Superior (1998: Paris, França). Tendências da educação superior para o século XXI. Brasilia: CRUB, 1999. 